\title{
Characterization of Function of Three Domains in Dishevelled-1：DEP Domain is Responsible for Membrane Translocation of Dishevelled-1
}

\author{
Wei Jun PAN ${ }^{1 *}$, Shu Zhao PANG ${ }^{1,2 *}$, Tao HUANG ${ }^{1}$, Hui Yun $\mathrm{GUO}^{2}$, Dianqing $\mathrm{WU}^{3}$, Lin $\mathrm{LI}^{1 * *}$ \\ ${ }^{1}$ Laboratory of Molecular Cell Biology, Institute of Biochemistry and Cell Biology, Shanghai Institutes for Biological Sciences, \\ Chinese Academy of Sciences, Graduate School of the Chinese Academy of Sciences, 320 Yueyang Rd, Shanghai 200031; \\ ${ }^{2}$ College of Life Science, Jilin University, Changchun 130061, China. \\ ${ }^{3}$ Department of Genetics and Developmental Biology, University of Connecticut Health Center, Farmington, Connecticut \\ 06030, USA.
}

\begin{abstract}
Wnt signaling plays an important role in embryogenesis and tumorgenesis. Although the mechanism about how Wnts transduce their signaling from receptor frizzled (Fz) to cytosol has not been understood, dishevelled (Dvl) protein was considered as the intersection of Wnt signal traffic. In this study, we characterized the function of three domains (DIX, PDZ and DEP) of Dvl-1 in canonical Wnt signal transduction and Dvl-1 membrane translocation. It was found both DIX and DEP domain were sufficient to block Wnt-3a-induced LEF-1 transcriptional activity and free cytosol $\beta$ catenin accumulation; whereas PDZ domain and a functional mutant form of DEP domain (DEP-KM) had no effect on canonical Wnt signaling. In addition, when cotransfected with Fz-7, DEP domain, but not DIX, PDZ or DEP-KM, translocated and co-localized with Fz-7 to the plasma membrane, which was similar to Dvl-1. Furthermore, it was DEP domain that could block Fz-7-induced membrane translocation of Dvl-1 via a possible competitive mechanism. These results strongly suggest that DEP domain is responsible for the membrane translocation of Dvl-1 protein upon Wnt signal stimulation.
\end{abstract}

Keywords: Wnt, frizzled-7, dishevelled, DIX, PDZ, DEP, membrane translocation, signal transduction

\section{INTRODUCTION}

Wnts are a family of secreted proteins involved in a wide range of developmental processes [1-4] and have been implicated in tumorigenesis $[5,6]$. Of the distinct Wnt pathways, the canonical Wnt signaling pathway, which involves the inhibition of GSK-3 $\beta$ activity and stabilization of $\beta$-catenin in the cytoplasm, is essential for the specification of cell fate. The noncanonical Wnt pathway, which involves the Rho family of GTPases and JNK, affects planar polarity in the same species [7]. And the noncanonical $\mathrm{Wnt} / \mathrm{Ca}^{2+}$ pathway stimulates intracellular calcium release in a $\mathrm{G}$ protein-dependent manner [8].

Wnt proteins have been shown to bind to members of the frizzled (Fz) family of serpentine receptors [9]. Until now, at least nine members have been identified in mammals [3]. All Fz proteins have an extracellular cysteine-rich

\footnotetext{
*These authors contributed equally to this work.

${ }^{* *}$ Correspondence: Lin LI

Tel: (86) 215492 1188, Fax: (86) 215492 1288,

E-mail: 1li@sibs.ac.cn
}

domain followed by seven putative transmembrane segments; their C-terminal cytoplasmic regions may differ significantly in length and in sequence similarity [3]. In canonical Wnt signaling, another co-receptor, LRP5/6 have been identified [10-13]. Canonical Wnt signaling is only transduced when both Fz and LRP are in complex with Wnt. By an unknown mechanism, Wnt binds to $\mathrm{Fz}$ and transduces signal to cytoplasmic dishevelled (Dvl) protein, leading to inhibition of the activity of GSK-3 $\beta$. Meanwhile, the scaffold protein of a $\beta$-catenin degradation complex-Axin translocates to the membrane where it interacts with the intracellular tail of LRP-5 [14]. By sequentially destabilizing Axin and inhibiting GSK- $3 \beta, \beta$-catenin becomes refractory to degradation. It accumulates in the cytoplasm, eventually translocates to the nucleus and activates TCF/ LEF-1 target genes [3]. Without canonical Wnt signal, $\beta$ catenin is always recruited and phosphorylated by multicomponent degradation complexes, including APC/Axin/ CKI- $\alpha /$ GSK $3 \beta$ and some PP2A subunits, and then undergo ubiquitylation and proteasomal breakdown [3].

Dvl proteins are composed of an N-terminal DIX domain, 
a central PDZ domain, and a C-terminal DEP domain [15, 16]. It acts downstream of $\mathrm{Fz}$ receptors both in planar polarity signalling and in segmental polarity of the epidermis in Drosophila, which are $\beta$-catenin independent and dependent, respectively [15,17-19]. Several lines of evidence suggest that Dvl activates distinct signalling pathways through distinct domains. For example, the $\mathrm{C}$ terminal DEP domain is required for the activation of JNK pathway, while DIX domain is essential for canonical Wnt pathway [15]. Dvl is phosphorylated both in vivo and in vitro in response to $\mathrm{Wnt}$ and $\mathrm{Fz}$ receptors. A functional DEP domain is required for the phosphorylation of Dvl, which is closely related to membrane relocalization, indicating that it is important for the function of Dvl in transducing signalings [19, 20].

To validate how Fz receptor talk with Dvl protein, Umbhauer et al identified a short conserved motif (LysThr-X-X-X-Trp) located two amino acids after the seventh transmembrane domain, which is required for activation of the Wnt/ $\beta$-catenin pathway and for membrane relocalization and phosphorylation of Dvl in Xenopus [21]. By using NMR spectroscopy, it was determined that an internal sequence of Fz weakly binds to the conventional peptide-binding site in the PDZ domain of Dvl [22]. However, this weak interaction is defined as a previously unknown connection between the membrane-bound receptor and downstream components of the Wnt signaling pathways. The mechanism of how Wnts transduce their signalings from Fz to cytoplasmic Dvl protein is still unclear. In this work, we demonstrated that DEP domain was critically involved in Fz7-induced translocation of Dvl-1 to plasma membrane.

\section{MATERIALS AND METHODS}

\section{Plasmid constructs}

The wild type and mutant forms of mouse Dvl-1, mouse Fz-7, human LEF-1, mouse $\beta$-catenin were generated by PCR using the high fidelity thermo-stable DNA polymerase, Pfu (Stratagene, CA). Myc, HA, or flag epitope tags, or GFP protein were introduced to the $\mathrm{C}$-termini of the full-length and mutant molecules. The expression of these molecules was driven by a CMV promoter [15, 23]. The LEF-1 reporter gene constructs were kindly provided by Dr. Grosschedl [24].

\section{Cell cultures}

Human embryonic kidney (HEK) 293 cells were maintained in Dulbecco's Modified Eagle Medium (DMEM) (Invitrogen) containing $10 \%$ fetal bovine serum (Hyclone). Wnt-3a-containing conditioned medium were prepared as previously described [14].

\section{Transfection and Reporter gene assay}

Transfection was carried out using Lipofectamine Plus as suggested by the manufacturer (Life Technologies, MD). The transfec- tion was stopped after $3 \mathrm{~h}$. For reporter gene assays, cell extracts were collected $24 \mathrm{~h}$ later. Cell lysates were measured for fluorescence intensity emitted by GFP proteins in a FL600 fluorometer (BIOTEK Inc.), and then the luciferase substrate (Boehringer Mannheim Luciferase Assay Kit) was added for determining the luciferase activities using a MicroLumate Plus luminometer (Perkin Elmer Inc.). Luminescence intensity is normalized against fluorescence intensity.

\section{Cell fraction}

Cells in 6-well plates were washed once with ice-cold PBS and scraped into hypotonic buffer containing $10 \mathrm{mM}$ HEPES ( $\mathrm{pH}$ 7.9), $1.5 \mathrm{mM} \mathrm{MgCl}_{2}, 1 \mathrm{mM}$ EDTA, $10 \mathrm{mM} \mathrm{KCl}, 0.5 \mathrm{mM}$ DTT and a cocktail of protease inhibitors (Boehringer). After put on ice for 10 min, cells were homogenized in a homogenizer, and then centrifuged at $15,000 \mathrm{~g}$. The supernatant was directly loaded by SDS sample buffer and saved as the cytosol fraction.

\section{Western blotting}

Samples were assayed by SDS-PAGE, and proteins were electroblotted to nitrocellulose membranes. Results were visualized using an imaging system with an infrared imaging system (LI-COR Biosciences Inc.). Mouse anti-HA and anti-myc (purchased from Bibco), anti- $\beta$-catenin (BD Transduction Laboratories), and anti- $\beta$ tubulin (Sigma) mono-antibodies were used to, respectively, detect various samples as described in the text.

\section{Immunofluorescence staining and Microscopy}

HEK 293 cells on coverslips were washed once with ice-cold PBS and then fixed for $30 \mathrm{~min}$ in PBS containing 4\% paraformaldehyde at $4{ }^{\circ} \mathrm{C}$. After the fixed cells were incubated in $0.2 \%$ Triton X-100 contained PBS at room temperature for $30 \mathrm{~min}$, coverslips were incubated with $1 \%$ BSA for $1 \mathrm{~h}$ and then with anti-HA antibody (1:100) for another $1 \mathrm{~h}$. Cy3-conjugated anti-mouse IgG (Jackson Immunoresearch Laboratories, PA) was used as the second antibody. Cells were examined using a fluorescence microscope (Leica TCS SP2).

\section{RESULTS}

\section{Effects of domains of Dvl-1 on the transcriptional activity of Wnt signaling}

The cytoplasmic protein Dvl and the receptor Fz are essential in canonical and noncanonical Wnt signaling pathways [25-30]. It is believed that Dvl molecule transmits the signal from plasma membrane, and decides which event(s) would occur in Wnt-responsive cells [31]. To demonstrate whether and how Dvl protein accepts would upstream signal and/or translocates to plasma membrane, we made four constructions for the three domains of mouse Dvl-1: Dvl-1-DIX (DIX, residues 1 200), Dvl-1PDZ (PDZ, residues 201 375), Dvl-1-DEP (DEP, residues 400 503), and a functional mutant of Dvl-1-DEP (DEP-KM, K434M mutation in electric dipole on the molecule's surface of DEP domain) [16]. The effects of these domains on the canonical Wnt pathway were determined. 


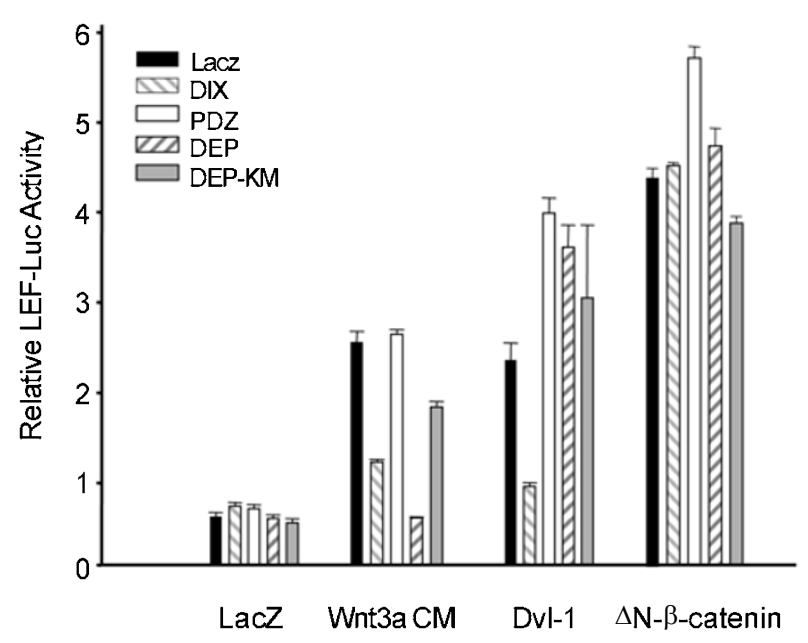

Fig. 1 DIX and DEP domain block Wnt-3a-induced LEF-1 reporter gene activity. HEK 293 cells in 24 -well plates were transfected with a combination of expression plasmid including $12.5 \mathrm{ng}$ of LEF-1, $37.5 \mathrm{ng}$ of LEF-1 luciferase reporter, $50 \mathrm{ng}$ of GFP, and $25 \mathrm{ng}$ of Dvl-1 or $7.5 \mathrm{ng} \Delta \mathrm{N}-\beta$-catenin in the absence or the presence of $50 \mathrm{ng}$ of DIX, PDZ, DEP, or DEP-KM, respectively. LacZ plasmid was added to ensure the amount of each transfected DNA was equal $(0.25 \mu \mathrm{g} /$ transfection $)$. After $18 \mathrm{~h}$, cells were treated with control medium or Wnt-3a conditioned-medium (CM) for additional $6 \mathrm{~h}$, then cells were lysed for GFP and luciferase activity assay as described in Materials and Methods. The luciferase activities were normalized against the levels of GFP expression. The error bar represents the standard deviation. All luciferase reporter gene assays were carried out in duplicate and repeated at least three times.

In LEF-1 reporter gene assay, Dvl-1 mutants were cotransfected with LEF-1 reporter gene, LEF-1, full-length Dvl-1, and $\Delta \mathrm{N}-\beta$-catenin into HEK 293 cells. After $18 \mathrm{~h}$, Wnt-3a conditioned-medium (CM) was added for an additional $6 \mathrm{~h}$ treatment, and then the luciferase activity was determined. As shown in fig. 1, either Wnt-3a CM or two downstream activators, Dvl-1 [16] and $\Delta \mathrm{N}-\beta$-catenin [32] could dramatically elevate the luciferase activity of LEF-1 reporter gene. Both DIX and DEP domain had a strong inhibitory effect on Wnt-3a-induced transcriptional activity, whereas PDZ domain alone almost did not have any effect (Fig. 1). Compared with DEP, the mutant DEP-KM suppressed the Wnt-3a-induced LEF activity in a much weaker manner. On the other hand, only DIX but not PDZ and DEP domain, inhibited the transcriptional activity induced by overexpressed Dvl-1 (Fig. 1). However, all these three domains did not have any effects on the LEF reporter activity induced by $\Delta \mathrm{N}-\beta$-catenin (Fig. 1).

$\Delta \mathrm{N}-\beta$-catenin was a constitutively active form of $\beta$ catenin. Due to the lack of N-terminal GSK-3 $\beta$ and CKI $\alpha$ phosphorylation sites, the protein level of this mutant

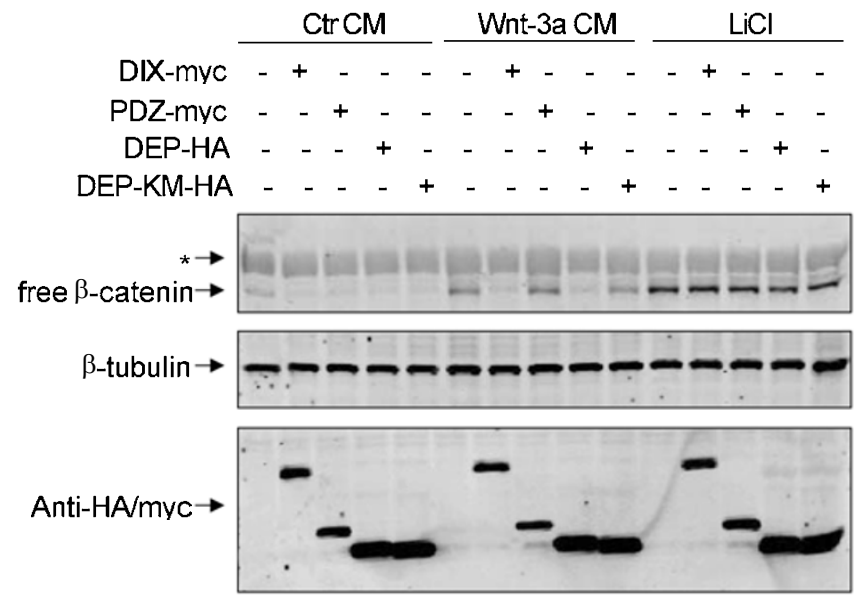

Fig. 2 Effects of domains of Dvl-1 on Wnt-3a/LiCl-induced free $\beta$ catenin accumulation. HEK 293 cells in 6-well plate were transfected with 200 ng DIX-myc, PDZ-myc, DEP-HA, or DEP-KM-HA. LacZ plasmid was added to make the total amount of DNA equal $(1.0 \mu \mathrm{g} /$ transfection). After $18 \mathrm{~h}$, cells were treated with control medium, Wnt-3a-conditioned-medium or $\mathrm{LiCl}(10 \mathrm{mM})$ as indicated for additional $6 \mathrm{~h}$. Then cells were harvested and cytosol fraction was prepared as described in Materials and Methods. Endogenous cytosol free $\beta$-catenin and ectopic expression Dvl fragments were analyzed by Western blotting. As a control, the expression of $\beta$-tubulin was also detected.

cannot be regulated by the upstream components of canonical Wnt pathway [32]. DEP and DIX domains only affect Wnt-induced, but not $\Delta \mathrm{N}-\beta$-catenin-induced LEF-1 transcriptional activity, indicating that these fragments of Dvl- 1 may regulate the process of $\beta$-catenin accumulation.

To verify if and how these Dvl-1 domains affect Wnt3a CM-induced free $\beta$-catenin accumulation, HEK 293 cells were transfected with various Dvl-1 domains, respectively. After $18 \mathrm{~h}$, cells were treated with Control CM, Wnt-3a $\mathrm{CM}$ or a chemical inhibitor of $\mathrm{GSK} 3 \beta, \mathrm{Li}^{+}(\mathrm{LiCl})$ [3] for additional $6 \mathrm{~h}$. Then the cells were harvested for preparation of cell fraction and Western blotting analysis. Quantified by Odyssey v1.2 software supported by LI-COR Biosciences Inc and normalized with the level of endogenoustubulin, the level of endogenous free cytosol $\beta$-catenin was found to be elevated in 4.8 (Wnt-3a CM) or $15.4(\mathrm{LiCl})$ folds respectively. Both DIX and DEP domain almost completely abolished the accumulation of free $\beta$-catenin induced by Wnt-3a, whereas DEP-KM had much weaker effect and PDZ had no effect (Fig. 2). These results are in 


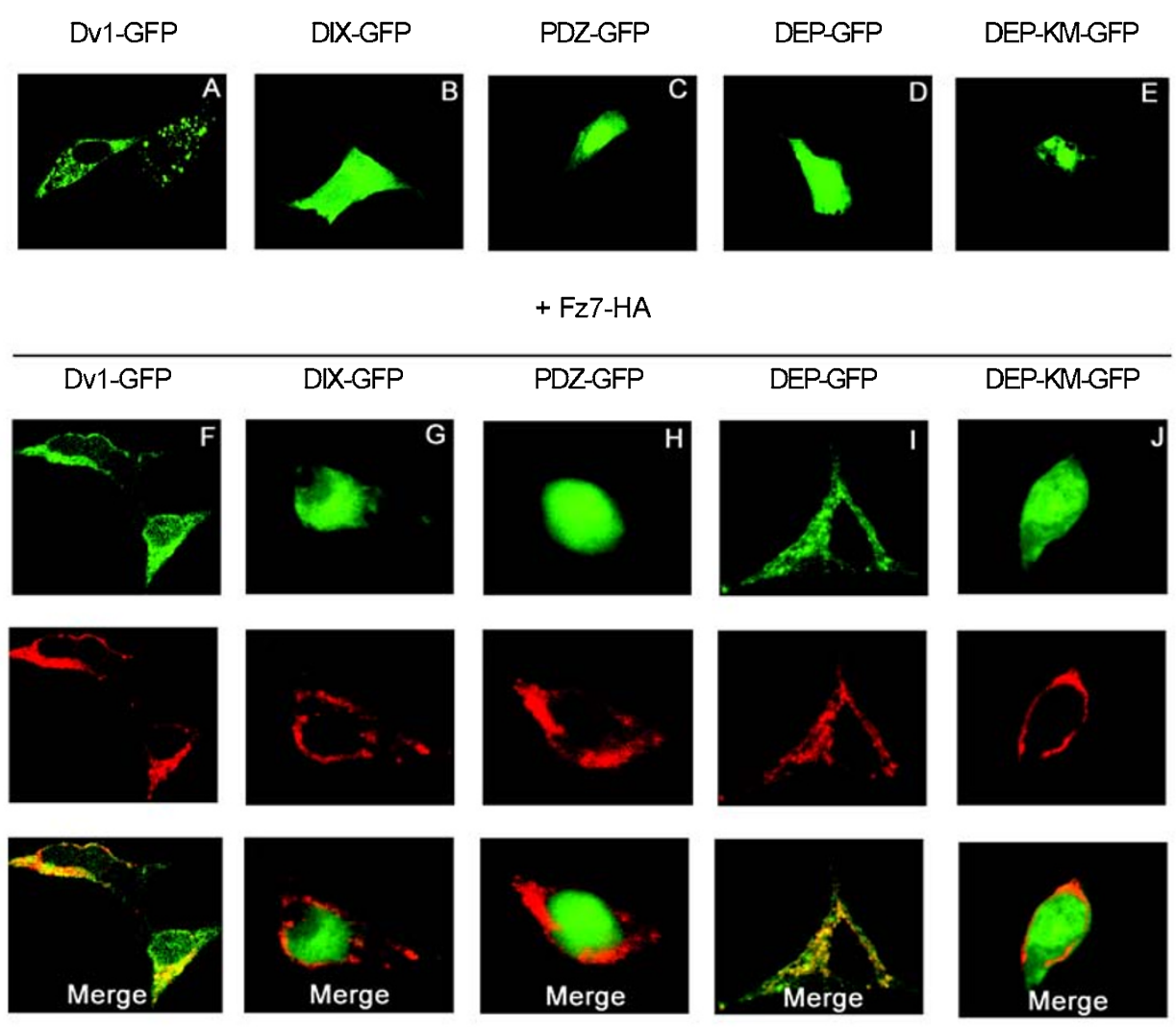

Fig. 3 Dvl-1 and its DEP domain translocates to plasma membrane with Fz7. HEK 293 cells in 35 mm dishes were co-transfected with 100 ng of (A, F) Dvl-1-GFP, (B, G) DIX-GFP, (C, H) PDZ-GFP, (D, I) DEP-GFP, (E, J) DEP-KM-GFP, and without (A-E) or with (F-J) 400 ng of Fz-7-HA. LacZ expression plasmid was added to make the total amount of DNA equal (1.0 $\mu \mathrm{g} /$ transfection). After $1 \mathrm{~d}$ culture on coverslips, cells were fixed for immunostaining assay. The GFP signal in green indicates the fusion protein and Fz-HA is shown in red.

consistent with the results found in LEF-1 reporter gene assay (Fig. 1). At the same time, none of Dvl-1 domains changed $\beta$-catenin accumulation (Fig. 2) and the transcriptional activity of LEF-1 reporter gene induced by $\mathrm{LiCl}$ (data not shown).

\section{DEP domain translocates to plasma membrane with Fz-7}

After the characterization of three domains of Dvl-1 in canonical Wnt signal pathway, we can briefly conclude that DIX domain and DEP domain have inhibitory influence on the Wnt signaling at the upstream of $\beta$-catenin and GSK, and that DEP domain obviously mediates the the talk upstream but not the downstream of Dvl-1. We were interested to further see whether DEP was involved in Dvl membrane translocation mediated by Wnt or Fz in mammalian cells.

Full-length Dvl-1 and its three domains were tagged with GFP protein and transfected into HEK 293 cells respectively. The distribution of GFP fusion form of Dvl-1 was restrictedly located in cytoplasm (Fig. 3A) and partially associated with cytosolic vesicle, which is similar to that of endogenous Dvl as previously reported [33]. DIX domain was also mainly distributed in the cytoplasm (Fig. 3B), but GFP-fused PDZ, DEP or DEP-KM was mainly localized in nuclei (Fig. 3C, D, E).

To test if $\mathrm{Fz}$ receptor could affect the distribution of Dvl-1 and its domains, all these GFP-fused proteins were respectively cotransfected with HA-tagged Fz-7 in HEK 293 cells. We found that none of these GFP-fused proteins could change the predominant membrane location of Fz-7 (Fig. 3F-J). As similar as its Xenopus ortholog, mouse Fz-7 induced the membrane translocation of Dvl-1 (Fig. $3 F)$. And it obviously showed a colocalization of Dvl-1 and Fz-7-HA. Interestingly, DEP domain was also colocalized with Fz-7 on plasma membrane (Fig. 3I). However, none of the GFP-fused DIX, PDZ, or K434M mutant of DEP could be translocated to cytoplasmic 
+ Dvl1-HA \& Fz7-flag
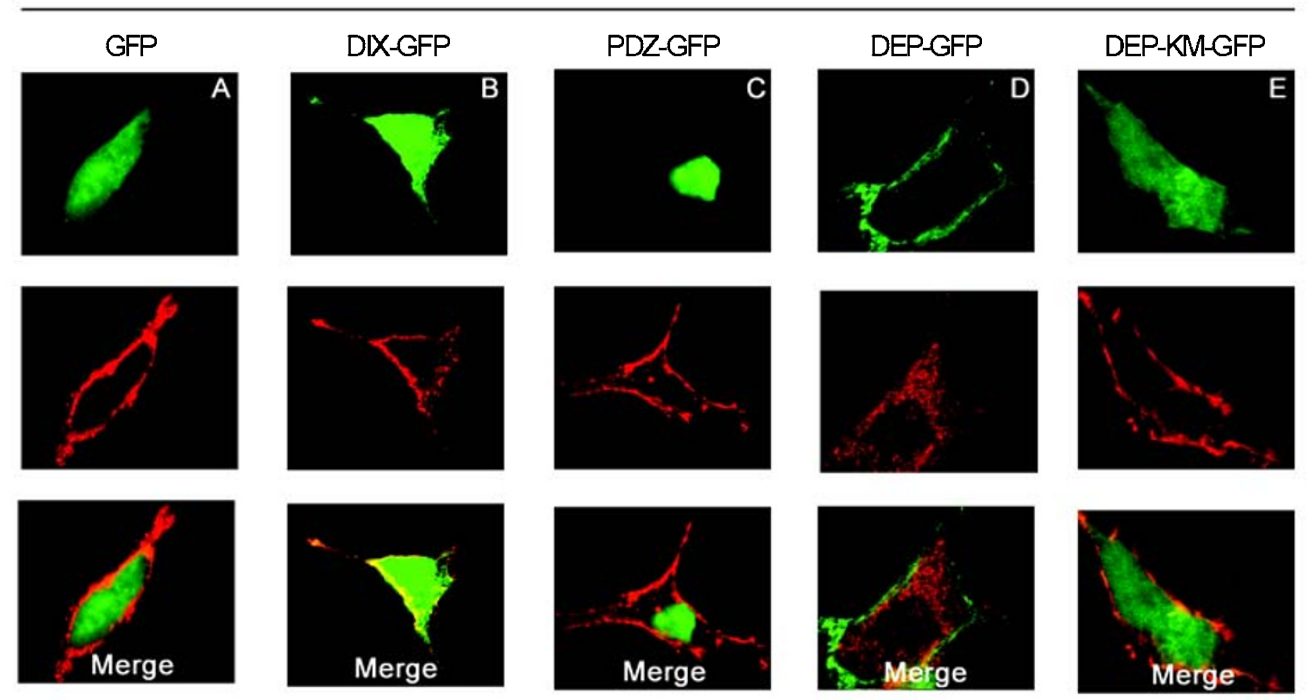

Fig. 4 DEP domain blocks membrane translocation of Dvl-1. HEK 293 cells in $35 \mathrm{~mm}$ dish were co-transfected with $100 \mathrm{ng}$ of Dvl-1-HA, $400 \mathrm{ng}$ of Fz-7-flag, and $100 \mathrm{ng}$ of (A) GFP, (B) DIX-GFP, (C) PDZ-GFP, (D) DEP-GFP, and (E) DEP-KM-GFP. LacZ expression plasmid was added to make the total amount of DNA equal $(1.0 \mu \mathrm{g} /$ transfection). After $1 \mathrm{~d}$ cultured on coverslips, cells were fixed for immunostaining assay. The GFP signal in green indicates the fusion protein and Fz-HA is shown in red.

membrane by or colocalized with Fz-7-HA (Fig. 3G, H, J).

\section{DEP domain attenuates Fz-7-induced Dvl-1mem- brane translocation}

Among the three domains of Dvl-1 only DEP was shown to be colocalized with Fz-7 as well as the full-length Dvl-1, suggesting that the DEP domain of Dvl-1 may be responsible for the colocalization of Dvl-1 and Fz-7. To give more evidences for this hypothesis, we further tested the effects of various domains of Dvl-1 on its translocation to the plasma membrane in the presence of transfected Fz-7 As shown in fig. 4, when cotransfected with Fz-7 into HEK 293 cells, Dvl-1-HA was localized on the plasma membrane, similar to Dvl-1-GFP when cotransfected with Fz-7-HA (Fig. 3F). As control, GFP alone has no effect on this colocalization (Fig. 4A). DEP domain, as shown in Fig. 4D, replaced the location of Dvl-1, and totally distributed around the plasma membrane, indicating that DEP could disrupt the colocalization of Dvl-1 with Fz-7 in the membrane. We suppose that this is due to a competitive colocalization with Fz-7 between DEP domain and Dvl-1 molecule. DIX domain could be visualized with an obviously colocalization with Dvl-1 on the membrane, but as same as PDZ domain, it could not affect the localization of Dvl-1 (Fig. 4B, C). Compared with DFP domain, DEP-KM could not effectively attenuate the membrane translocation of Dvl-1 (Fig. 4E).

\section{DISCUSSION}

Highly conserved signaling protein Dvl is required for the transduction and diversity of Wnt signaling. Genetic analysis in Drosophila melanogaster has shown that Dvl plays an important role in both cell fate determination and cell polarity [31]. It is demonstrated that $\mathrm{Fz}$ transduces the canonical Wnt signaling from membrane to cytoplasm via a Dvl-dependent manner, even if the canonical Wnt signal from co-receptor LRP-6 is transduced via a Dvlindependent manner [23]. The overexpresssion of some Fz proteins in Xenopus could lead to the duplication of D-V Axis, and the membrane translocation of Dvl could be detected at the same time [21]. Beyond canonical Wnt pathway, genetic evidence suggests that the prototypic Drosophila Fz-1 is required for the establishment of tissue polarity through the JNK-1 pathway via Dvl and RhoA, and the membrane translocation of Dvl is required for this process [17, 34-37]. Similarly, vertebrate Fz homologues have also been shown to activate noncanonical Wnt pathway and induce the membrane translocation of Dvl (Fig. $3 \mathrm{~A}$, and unpublished data). The overexpression of Dvl and these $\mathrm{Fz}$ proteins could also induce JNK activation and $\mathrm{Ca}^{2+}$ signaling in mammalian cells [38,39, and unpublished data]. These results suggest that the membrane translocation of Dvl may take part in more pathways besides canonical Wnt signaling.

Previously, Wong et al [22] reported that DEP-contain- 
ing mutants inhibit Wnt-induced, but not Dvl-induced activation of the transcription factor LEF-1, as we showed in this study and before [23]. NMR structure of DEP domain suggests that this domain could interact with regulators upstream of Dvl via a strong electric dipole on the molecule's surface created by K434, D445 and D448 [16]. A Dvl mutant, lacking the C-terminal DEP domain, could not enhance LEF-1 reporter gene activity, unless it was added with a myristate signal (unpublished data). A myristate shorter form of Dvl, but not DIX domain alone, has also been reported to activate canonical Wnt signaling [40]. These results strongly suggest that DEP domain is critically involved in receiving the signaling from membrane.

In this work, we found that mammalian Dvl as well as its DEP domain could be translocated to plasma membrane when cotransfected with mouse Fz-7 (Fig. 3I). This membrane location of DEP could compete with Fz-7-induced membrane localization of full-length Dvl in mammalian cells (Fig. 4D). And these functions of DEP domain were dramatically weakened by its K434M mutation (Fig. 3 $\mathrm{J}, 4 \mathrm{E}$ ), which also show a weak inhibitory effect on Wnt3ainduced transcriptional activity and free $\beta$-catenin accumulation (Fig. 1 and 2). Here we could briefly conclude that DEP domain is responsible for membrane translocation of Dvl-1. It is very interesting to see if there is a direct interaction between $\mathrm{Fz}-7$ and DEP domain, and such work is in progress.

Although both DIX domain and PDZ domain were verified to be critical for Dvl in the transduction of canonical Wnt signaling $[15,41]$, none of them could be relocalized to plasma membrane by overexpressed Fz-7 (Fig. 3G, H), or influence $\mathrm{Fz}-7$-induced membrane translocation of Dvl-1 (Fig. 4B, C). Because DIX domain obviously inhibits the transcriptional activity of LEF-1 induced by Wnt3a CM and overexpressed Dvl-1, its function should be defined at the downstream of Dvl. Colocalization of DIXGFP with Dvl-1 on membrane which is induced by Fz-7 (Fig. 4B) suggests that dimerization of Dvl has no effect on its membrane location. Although the physical association of PDZ domain and C-terminal motif of Fz were discovered recently [22], we did not see any effects of PDZ domain alone on the Wnt-induced LEF-1 transcriptional activity (Fig. 1) or the colocalization of Fz and Dvl-1 (Fig. 3H, 4C). We suppose that the function of PDZ is not to decide the membrane translocation of Dvl, but its binding to $\mathrm{Fz}$ in a weak affinity may help to distinguish the distinct Wnt signaling.

\section{AKCNOWLEDGEMENT}

We thank Xiao Qing GAN and Wei BIAN for technical help. This work was supported by grants from the National
High Technology Research and Development Program of China (2003AA221050), the Major State Basic Research Development program of China (2002CB513000), National Natural Science Foundation of China and Chinese Academy of Sciences.

\section{Received, May 27, 2004}

Revised, Jun 18, 2004

Accepted, Jun 20, 2004

\section{REFERENCES}

1 Moon RT, DeMarais A, Olson DJ. Responses to Wnt signals in vertebrate embryos may involve changes in cell adhesion and cell movement. J.Cell Sci.Suppl 1993; 17:183-8.

2 Cadigan KM, Nusse R. Wnt signaling: a common theme in animal development. Genes Dev. 1997; 11:3286-305.

3 Wodarz A, Nusse R. Mechanisms of Wnt signaling in development. Annu.Rev.Cell Dev.Biol. 1998; 14:59-88.

4 Moon RT, Bowerman B, Boutros M, Perrimon N. The promise and perils of Wnt signaling through beta-catenin. Science 2002; 296:1644-6.

5 Taipale J, Beachy PA. The Hedgehog and Wnt signalling pathways in cancer. Nature 2001; 411:349-54.

6 Polakis P. Wnt signaling and cancer. Genes Dev 2000; 14:183751.

7 Veeman MT, Axelrod JD, Moon RT. A second canon. Functions and mechanisms of beta-catenin-independent Wnt signaling. Dev Cell 2003; 5:367-77.

8 Kuhl M, Sheldahl LC, Park M, Miller JR, Moon RT. The Wnt/ $\mathrm{Ca}^{2+}$ pathway: a new vertebrate $\mathrm{Wnt}$ signaling pathway takes shape. Trends Genet 2000; 16:279-83.

9 Yang-Snyder J, Miller JR, Brown JD, Lai CJ, Moon RT. A frizzled homolog functions in a vertebrate Wnt signaling pathway. Curr Biol 1996; 6:1302-6.

10 Tamai K, Semenov M, Kato Y, Spokony R, et al. LDL-receptorrelated proteins in Wnt signal transduction. Nature 2000; 407: 530-5.

11 Pinson KI, Brennan J, Monkley S, Avery BJ, Skarnes WC. An LDL-receptor-related protein mediates Wnt signalling in mice. Nature 2000; 407:535-8.

12 Wehrli M, Dougan ST, Caldwell K, et al. arrow encodes an LDLreceptor-related protein essential for Wingless signalling. Nature 2000; 407:527-30.

13 Mao B, Wu W, Li Y, et al. LDL-receptor-related protein 6 is a receptor for Dickkopf proteins. Nature 2001; 411:321-5.

14 Mao J, Wang J, Liu B, et al. Low-density lipoprotein receptorrelated protein- 5 binds to Axin and regulates the canonical Wnt signaling pathway. Mol Cell 2001; 7:801-9.

15 Li L, Yuan H, Xie W, et al. Dishevelled proteins lead to two signaling pathways. Regulation of LEF-1 and c-Jun N-terminal kinase in mammalian cells. J Biol Chem 1999; 274:129-34.

16 Wong HC, Mao J, Nguyen JT, et al. Structural basis of the recognition of the dishevelled DEP domain in the Wnt signaling pathway. Nat Struct Biol 2000; 7:1178-84.

17 Boutros M, Paricio N, Strutt DI, Mlodzik M. Dishevelled activates $\mathrm{JNK}$ and discriminates between JNK pathways in planar polarity and wingless signaling. Cell 1998; 94:109-18.

18 Moriguchi T, Kawachi K, Kamakura S, et al. Distinct domains of 
mouse dishevelled are responsible for the c-Jun N-terminal kinase/stress-activated protein kinase activation and the axis formation in vertebrates. J Biol Chem 1999; 274:30957-62.

19 Rothbacher U, Laurent MN, Blitz IL, et al. Functional conservation of the Wnt signaling pathway revealed by ectopic expression of Drosophila dishevelled in Xenopus. Dev Biol 1995; 170: 717-21.

20 Tada M, Smith JC. Xwnt11 is a target of Xenopus Brachyury: regulation of gastrulation movements via Dishevelled, but not through the canonical Wnt pathway. Development 2000; 127: 2227-38.

21 Umbhauer M, Djiane A, Goisset C, et al. The C-terminal cytoplasmic Lys-thr-X-X-X-Trp motif in frizzled receptors mediates Wnt/beta-catenin signalling. EMBO J 2000; 19:4944-54.

22 Wong HC, Bourdelas A, Krauss A, et al. Direct binding of the PDZ domain of Dishevelled to a conserved internal sequence in the C-terminal region of Frizzled. Mol Cell 2003; 12:1251-60.

23 Li L, Mao J, Sun L, Liu W, Wu D. Second cysteine-rich domain of Dickkopf-2 activates canonical Wnt signaling pathway via LRP-6 independently of dishevelled. J Biol Chem 2002; 277: 5977-81.

24 Hsu SC, Galceran J, Grosschedl R. Modulation of transcriptional regulation by LEF-1 in response to Wnt-1 signaling and association with beta-catenin. Mol Cell Biol 1998; 18:4807-18.

25 Wodarz A, Nusse R. Mechanisms of Wnt signaling in development. Annu Rev Cell Dev Biol 1998; 14:59-88.

26 Giles RH, van Es JH, Clevers H. Caught up in a Wnt storm: Wnt signaling in cancer. Biochim Biophys Acta 2003; 1653:1-24.

27 Veeman MT, Axelrod JD, Moon RT. A second canon. Functions and mechanisms of beta-catenin-independent Wnt signaling. Dev Cell 2003; 5:367-77.

28 Nusse R. Wnts and Hedgehogs: lipid-modified proteins and similarities in signaling mechanisms at the cell surface. Development 2003; 130:5297-305.

29 Molenaar M, Destree O. A tight control over Wnt action. Int J Dev Biol 1999; 43:675-80.
30 Shulman JM, Perrimon N, Axelrod JD. Frizzled signaling and the developmental control of cell polarity. Trends Genet 1998; 14: 452-8.

31 Wharton KA, Jr.. Runnin' with the Dvl: proteins that associate with Dsh/Dvl and their significance to Wnt signal transduction. Dev Biol 2003; 253:1-17.

32 Ishitani T, Ninomiya-Tsuji J, Nagai S, et al. The TAK1-NLKMAPK-related pathway antagonizes signalling between betacatenin and transcription factor TCF. Nature 1999; 399:798802.

33 Capelluto DG, Kutateladze TG, Habas R, et al. The DIX domain targets dishevelled to actin stress fibres and vesicular membranes. Nature 2002; 419:726-9.

34 Adler PN, Krasnow RE, Liu J. Tissue polarity points from cells that have higher Frizzled levels towards cells that have lower Frizzled levels. Curr Biol 1997; 7:940-9.

35 Mlodzik M. Planar polarity in the Drosophila eye: a multifaceted view of signaling specificity and cross-talk. EMBO J 1999; 18:6873-9.

36 Boutros M, Mlodzik M. Dishevelled: at the crossroads of divergent intracellular signaling pathways. Mech Dev 1999; 83:27-37.

37 Boutros M, Mihaly J, Bouwmeester T, Mlodzik M. Signaling specificity by Frizzled receptors in Drosophila. Science 2000; 288:1825-8.

38 Sheldahl LC, Slusarski DC, Pandur P, et al. Dishevelled activates Ca2+ flux, PKC, and CamKII in vertebrate embryos. J Cell Biol 2003; 161:769-77.

39 Maye P, Zheng J, Li L, Wu D. Multiple mechanisms for Wnt11mediated repression of canonical Wnt signaling pathway. J Biol Chem 2004; 279:24659-65

40 Cong F, Schweizer L, Varmus H. Casein kinase Iepsilon modulates the signaling specificities of dishevelled. Mol Cell Biol 2004; 24:2000-2011.

41 Li L, Yuan H, Weaver CD, et al. Axin and Frat1 interact with dvl and GSK, bridging Dvl to GSK in Wnt-mediated regulation of LEF-1. EMBO J 1999; 18:4233-4240. 